\title{
RÉTENTION DES OVULES APRÈS OVULATION DANS LA CAVITÉ OVARIENNE DE LA CARPE HERBIVORE : COMPOSITION DES OVULES ET CAPACITÉ DE DÉVELOPPEMENT DES OEUFS ET DES ALEVINS.
}

\author{
M. KHARROUBI (1), M. DROUSSI (2), A. BADRI (1), A. BOUZIDI (3), \\ E. ELBOUSTANI (4)
}

(1) Lab. d'Hydrobiologie, Dépt. de Biologie, Fac. des Sciences Semlalia, B.P. 2390, MARRAKECH, 40001 Maroc, e-mail : kharroubi2@yahoo.fr

(2) Station de Carpiculture de la Deroua, B.P. 64, BENI MELLAL, Maroc.

(3) Lab. des Sciences de l'Environnement, Fac. des Sciences et Techniques, Dépt. de Biologie, B.P. 57, SETTAT, Maroc, e-mail : bouzidi@uh1.ac.ma

(4) Lab. de Biochimie nutritionnelle, Dépt. de Biologie, Fac. des Sciences Semlalia, B.P. 2390, MARRAKECH, 40001 Maroc.

\section{RÉSUMÉ}

Pour étudier l'effet de rétention prolongée des ovules dans la cavité ovarienne (in vivo) de la carpe herbivore, des extractions d'ovules de 15 femelles ( 3 à $5,6 \mathrm{~kg}$ ) ont eu lieu à différents intervalles de temps après ovulation (0,30,60, 90 et $120 \mathrm{~min})$. Le taux de fécondation, le taux de survie embryonnaire, le taux d'éclosion et la croissance en longueur totale des alevins jusqu'à 5 semaines après éclosion $(L 1=$ Longueur totale mesurée 2 semaines après éclosion, L2 = Longueur totale mesurée 3 semaines après éclosion, L3 = Longueur totale mesurée 4 semaines après éclosion, L4 = Longueur totale mesurée 5 semaines après éclosion) sont déterminés pour chaque temps d'extraction des ovules. II n'y a pas eu de différences significatives entre ces paramètres biologiques observés juste après ovulation et ceux observés 120 min après ovulation.

Sont déterminées également quelques caractéristiques de la composition des ovules à savoir la teneur en eau, le poids sec, les cendres brutes, le fer, le calcium, les protéines totales, les lipides totaux, les phospholipides et les sucres. Ces composés demeurent constants quand les ovules sont maintenus au sein de la femelle pour deux heures après ovulation.

Chez la carpe herbivore, la rétention post ovulatoire des ovules pendant 2 heures in vivo n'a pas d'effet ni sur leur composition biochimique examinée, ni sur la capacité de développement des œufs ni sur la croissance des alevins en terme de longueur totale. L'absence de changements des constituants des ovules pourrait expliquer la maintenance 
de la viabilité des œufs et celle de la croissance des alevins. Cependant, il importe de signaler une certaine différence de cette capacité de développement des œufs et des alevins entre les femelles reproduites ( $p<0,05$ pour chaque paramètre examiné).

Mots-clés : carpe herbivore (Ctenopharyngodon idella), rétention in vivo, postovulatoire, qualité des ovules, composition des ovules, viabilité des œufs, longueur totale des alevins.

\title{
GRASS CARP OVA RETENTION IN THE BODY CAVITY AFTER OVULATION: OVA COMPOSITION AND DEVELOPMENT CAPABILITY OF EGGS AND ALEVINS.
}

\begin{abstract}
To investigate the effects of allowing the eggs to be retained by the grass carp within the abdominal cavity after ovulation, eggs of 15 females $(3-5.6 \mathrm{~kg})$ were obtained at increasing periods of time after ovulation $(0,30,60,90$ and $120 \mathrm{~min})$.

For each time of ova stripping, the fertilization rate, the embryonic survival rate, the hatching rate and the total length of alevins until 5 weeks after hatching $(\mathrm{L} 1=$ total length 2 weeks after hatching, L2 $=$ total length 3 weeks after hatching, L3 $=$ total length 4 weeks after hatching, $L 4=$ total length 5 weeks after hatching) are determined. There was no significant differences between those biological parameters recorded just after ovulation and those recorded $120 \mathrm{~min}$ after ovulation.

Ova were analyzed for dry weight, water, ashes and levels of total proteins, total lipids, phospholipides, carbohydrates, calcium and iron. The above aspects of egg composition remained constant when the ova are retained in the abdominal cavity for 2 hours after ovulation.

These results indicate that the in vivo retention of grass carp ova for 2 hours after ovulation has no effect neither on their biochemical composition nor on egg viability, nor on embryonic survival nor on hatching nor on the growth (total length of alevins until 5 weeks after hatching). The lack of changes in the composition of ova during their retention in ovario may explain that we still obtain satisfactory rates of viability 2 hours after ovulation and that the growth of alevins is not influenced. However, the composition of ova, the developmental capability of eggs and the growth of alevins show a high variability between females ( $p<0.05$ for each investigated parameter).
\end{abstract}

Keys-words : grass carp (Ctenopharyngodon idella), in vivo retention, ova quality, egg viability, ova composition, total length of alevins.

\section{INTRODUCTION}

En pisciculture, le vieillissement des ovocytes ovulés est un facteur important à connaître lorsque la ponte est induite par traitement hormonal et le vieillissement est rapide comme dans le cas de la carpe commune et des carpes chinoises (HORVATH, 1978a ; SUZUKI, 1980). Le pisciculteur doit connaître avec précision le moment de l'ovulation et il doit aussi avoir une idée du temps dont il dispose ensuite pour la récolte et la fécondation des ovocytes. 
Jusqu'à aujourd'hui, les facteurs et les mécanismes gouvernant le vieillissement in vivo restent très peu abordés chez les cyprinidés comme chez les autres téléostéens. Ainsi, les changements biochimiques et chimiques ayant lieu au cours de la rétention des ovules in vivo ont été très peu étudiés. Cependant, il a été démontré que le séjour prolongé des ovules in vivo après ovulation induit des changements biochimiques importants au niveau des ovules et affecte ainsi les différents taux de viabilité de la progéniture (SAKAl et al., 1975 ; SUZUKI, 1975 ; HIROSE et al., 1979 ; STATOVA et al., 1982 ; BILLARD et al., 1986). II est à indiquer que durant le stockage des ovules in vivo et sous des conditions en anaérobie, l'acide lactique s'accumule et le $\mathrm{pH}$ diminue. Par conséquent, l'intégrité de l'enveloppe vitelline baisse suite à un réarrangement des lipides et protéines structuraux. Dans les ovaires, l'eau provenant du fluide ovarien entre dans les ovules provoquant leur collaps (ZHUKINSKIY et KIM, 1980). En effet, il est recommandé de féconder les ovules juste après ovulation. Cependant, en pratique, lors des opérations de reproduction artificielle à la station de la Deroua (Maroc), les femelles injectées arrivent à ovulation en même temps et on est contraint de différer le stripping (l'extraction des ovules) et la fécondation des ovules de certaines femelles. En effet, la manipulation de chaque femelle dure 15 à 20 minutes (anesthésie du poisson, extraction des ovules et fécondation) et le temps qui sépare la fécondation des ovules de la première femelle et ceux de la dernière femelle peut aller jusqu'à 120 minutes. Pendant cet intervalle de temps, les ovules seraient susceptibles de vieillir dans l'abdomen du poisson. Ce travail vise à apprécier les effets éventuels du vieillissement des ovules soumis à la rétention dans la cavité ovarienne en terme de taux de fécondation, de survie embryonnaire, d'éclosion et en terme de croissance en longueur totale des alevins. Le deuxième objectif de ce travail est d'étudier les effets des retards dans l'extraction des ovules sur quelques aspects de la composition des ovules.

\section{MATÉRIEL ET MÉTHODES}

Cette étude a été réalisée à l'écloserie de la station de carpiculture de Béni Mellal (Maroc), sur 15 femelles de carpe herbivore Ctenopharyngodon idella Valenciennes, durant les saisons de reproduction des années 1996, 1997 et 1998.

\section{Géniteurs et injections hormonales}

Les géniteurs de la carpe herbivore sont élevés dans des étangs en terre de $2000 \mathrm{~m}^{2}$ et ils sont alimentés à satiété à la luzerne verte. Les géniteurs matures sélectionnés sont transférés à l'écloserie et mis en stabulation dans des bacs circulaires de $1 \mathrm{~m}^{3}$ chacun. Après acclimatation, pesée et marquage, les géniteurs reçoivent la première dose d'extraits hypophysaires de la carpe commune (Agent Chemical Company). Les femelles reçoivent $0,3 \mathrm{mg} / \mathrm{kg}$ du poids vif dans une première injection et $3 \mathrm{mg} / \mathrm{kg}$ dans une deuxième injection pratiquée 12 heures après la première injection. Les mâles ne reçoivent qu'une seule injection à raison de $3 \mathrm{mg} / \mathrm{kg}$ lors de la première injection des femelles.

Grâce à l'injection hormonale, les ovules matures quittent les follicules et s'accumulent dans l'ovaire. En se basant sur les abaques établis par HORVATH (1978a), la sommation des températures notées à chaque heure après la première injection permet de déterminer sensiblement l'heure de ponte des femelles. L'ovulation de la carpe herbivore a lieu à 200-220 degrés-heures après la première injection. 


\section{Collecte des gamètes, fécondation et incubation}

Environ une demi heure avant l'ovulation des femelles, les mâles sont anesthésiés dans une solution de 200 p.p.m. de phénoxy-2-éthanol. Leur laitance est recueillie dans des tubes à essai secs. La mobilité des spermatozoïdes est examinée sous microscope.

Si la femelle est prête à ovuler, une légère pression sur la partie postérieure du ventre laisse écouler librement les ovules. On veille à ne pas appliquer une forte pression sur le ventre, autrement la femelle éjecte une partie de ces ovules dans le bac. La femelle est alors anesthésiée dans une solution de 200 p.p.m. de phénoxy-2-éthanol. Après expulsion à $22-24^{\circ} \mathrm{C}$, les ovules sont pesés et fécondés à sec par la laitance de 2 à 3 mâles. Un volume de $10 \mathrm{ml}$ de laitance peut féconder $1 \mathrm{~kg}$ d'ovules. Pour assister la fécondation, une solution fertilisante à base d'urée et de chlorure de sodium ( $3 \mathrm{~g}$ d'urée, $4 \mathrm{~g} \mathrm{NaCl}, 1$ I d'eau distillée) est versée sur le mélange des produits sexuels à raison de $20 \%$ de leur volume et les œufs sont rincés avec de l'eau fraîche après agitation délicate.

Immédiatement après hydratation, chaque lot d'œufs est maintenu dans deux incubateurs coniques de 30 litres de contenance chacun à une température de 22 à $24^{\circ} \mathrm{C}$.

On immerge la pipette de $25 \mathrm{ml}$ dans l'incubateur, les œufs, les embryons ou les alevins remontent par capillarité dans la pipette. Celle-ci traverse toute la colonne d'eau dans l'incubateur et permet ainsi d'obtenir un échantillon homogène et représentatif du lot d'œufs incubés. Pour améliorer la reproductibilité, quatre échantillons sont prélevés dans chaque incubateur pour déterminer chaque taux de viabilité.

\section{Rétention des ovules in vivo}

Pour étudier l'effet de la rétention des ovules in vivo sur la viabilité de la progéniture et sa croissance (taux de fécondation, taux de survie embryonnaire, taux d'éclosion et longueur totale des alevins), le protocole expérimental suivant a été adopté.

Cette étude a concerné les ovules provenant de 15 femelles, dont le poids a varié entre 3 et $5,6 \mathrm{~kg}$. Au moment de l'ovulation, un lot d'ovules de 100 à $150 \mathrm{~g}$ est extrait puis fécondé comme il a été décrit plus haut. L'extraction des autres lots d'ovules a lieu toutes les 30 minutes. Le séjour prolongé des ovules in vivo dans la cavité abdominale est assuré par la couture de l'orifice génital après chaque extraction.

\section{Détermination des taux de fécondation, de survie embryonnaire et d'éclosion}

L'incubation des œufs de la carpe herbivore dure 28 à 36 heures à une température de 20 à $24^{\circ} \mathrm{C}$. Huit heures après incubation, la segmentation du mésoderme débute. A ce stade, les ovules fécondés et non fécondés sont faciles à reconnaître sous loupe binoculaire. Le Taux de Fécondation (TF) est déterminé $8 \mathrm{~h}$ après fécondation en comptant le nombre d'œufs développés et celui des œufs non développés. Le TF est défini comme étant : (nombre des ovules fécondés / nombre total des ovules) x 100.

Le Taux de Survie Embryonnaire (TSE) est déterminé $24 \mathrm{~h}$ après fécondation. A ce stade, les embryons viables sont reconnaissables à leur yeux noirs et à leur somites alors que les embryons qui ont avorté représentent une masse blanche allongée sur le vitellus et qui a commencé à se désintégrer. Le TSE est défini comme étant : (nombre des embryons viables / nombre total des embryons) $\times 100$.

Le Taux d'Eclosion (TE) est déterminé $36 \mathrm{~h}$ après fécondation. Les embryons qui n'ont pas éclos restent séquestrés dans leur coquilles. Le TE est défini comme étant : (nombre des embryons qui ont éclos / nombre total des embryons du stade $36 \mathrm{~h}$ ) $\times 100$. 


\section{Suivi de la croissance en longueur totale des alevins}

Les larves qui viennent d'éclore (500 de chaque lot) sont mises dans des containers en tissus. Ces containers sont tenus dans des aquariums ayant une capacité d'alevinage de 300000 larves par aquarium. Trois jours après éclosion, le vitellus est résorbé et le poisson reçoit une solution d'œuf de poule à raison de $2 \mathrm{ml}$ par container toutes les trois heures. Vigoureux après ce séjour prolongé dans l'écloserie, cent alevins de chaque lot sont transférés à l'étang. Ils se nourrissent de la production naturelle de l'étang (zooplancton) et reçoivent une alimentation exogène à base de farine de poisson (55\% de protéines) à raison de $2 \mathrm{~g}$ pour 100 alevins.

Dès que les alevins achèvent leur première semaine à l'étang, leur longueur totale est mesurée in situ sur un échantillon de 10 individus par container. Pendant un mois, chaque semaine, une mesure de longueur totale de la fraie de chaque container est relevée $(\mathrm{L} 1=$ Longueur totale mesurée 2 semaines après éclosion, $\mathrm{L} 2=$ Longueur totale mesurée 3 semaines après éclosion, L3 $=$ Longueur totale mesurée 4 semaines après éclosion, L4 = Longueur totale mesurée 5 semaines après éclosion).

\section{Composition des ovules}

Après chaque stripping, deux échantillons d'ovules ont été conservés à $-30^{\circ} \mathrm{C}$. Les composants étudiés sont exprimés en pourcentage du poids sec, exception faite pour le poids sec et l'eau en pourcentage du poids frais, pour le fer en pourcentage du poids $\sec \times 10^{-3}$ et pour les phospholipides exprimés par rapport aux lipides totaux.

Les échantillons de $100 \mathrm{mg}$ de poids frais d'ovules sont séchés à l'étuve pendant 48 heures à $105^{\circ} \mathrm{C}$ jusqu'à poids constant (AOAC, 1970) pour déterminer leur teneur en eau et leur poids sec.

La minéralisation a lieu dans un four à moufle à $550^{\circ} \mathrm{C}$ pendant 24 heures jusqu'à ce que les cendres soient blanches et exemptes de carbone puis le poids des cendres brutes est relevé.

La dissolution du résidu s'effectue par $5 \mathrm{ml}$ d'acide perchlorique $60 \%$ à $200^{\circ} \mathrm{C}$ pendant 10 minutes. Pour le dosage de fer, les minéralisats sont passés dans un appareil d'absorption atomique à flamme (type Unicam, 929) alors que pour celui du calcium, les minéralisats sont passés dans un spectrophotomètre à flamme (type Jenway).

La teneur en azote a été déterminée par microanalyse Kjeldahl (AOAC, 1970). Les $100 \mathrm{mg}$ séchés des échantillons à doser ont été minéralisés par l'acide sulfurique concentré en présence de catalyseur. La distillation de l'ammoniac à été effectuée dans un appareil Gerhardt. Le contenu protéique est obtenu en multipliant le contenu en azote par 6,25 .

Les lipides sont extraits selon la méthode de FOLCH et al. (1957) par le chloroforme-méthanol, $(2: 1 \mathrm{v} / \mathrm{v})$ contenant le Butyle hydroxytoluène à $5 \%$. Après agitation et ajout du $\mathrm{KCl}$, la préparation est centrifugée pendant $5 \mathrm{~min}$ à $3000 \mathrm{~g}$. La phase organique est prélevée puis filtrée sur le sulfate de sodium anhydre. Après évaporation à sec, le résidu est repris 3 fois par $100 \mu$ du mélange chloroforme-méthanol (1:1) dans un tube à vis préalablement taré. Une deuxième évaporation sous azote à sec est effectuée et le tube contenant le résidu est repesé pour déterminer le poids des lipides totaux.

Le dosage des phospholipides est effectué selon la technique de BARTLETT (1959). $10 \mu \mathrm{l}$ d'extrait lipidique est additionné du nitrate de magnésium $(0,42 \%)$ puis chauffé à flamme jusqu'à disparition des fumées rousses. Le minéralisat est récupéré dans $1 \mathrm{ml}$ de $\mathrm{HCl}$ puis maintenu dans un bain marie bouillant pendant 15 minutes. $1 \mathrm{ml}$ du mélange 
réactif est ajouté après refroidissement puis la préparation est incubée à $45^{\circ} \mathrm{C}$ pendant 20 minutes. Après l'apparition d'une couleur bleue, on lit au spectrophotomètre la densité optique à $820 \mathrm{~nm}$. La concentration du phosphore est déterminée à l'aide d'une courbe étalon $(D O=f($ concentration du phosphore $\mathrm{mg} / \mathrm{l})$ ). La concentration des phospholipides est égale à 25 fois celle du phosphore.

Le contenu en sucre est déterminé par la méthode colorimétrique utilisant le réactif acide sulfurique-phénol (DUBOIS et al., 1956).

\section{Méthodes statistiques}

L'analyse de variance à un seul facteur (I'ANOVA I) a été appliquée pour évaluer l'effet de la rétention des ovules in vivo $(30,60,90$ et 120 min après ovulation) sur le taux de fécondation, le taux de survie embryonnaire, le taux d'éclosion, sur la croissance en longueur totale des alevins et sur chaque paramètre examiné de la composition des ovules.

Les corrélations entre les différents indicateurs de viabilité (taux de fécondation, taux de survie embryonnaire et taux d'éclosion juste après ovulation et dans les conditions de stockage in vivo) sont recherchées par l'analyse de régression linéaire.

De la même manière sont recherchées les corrélations entre le poids des femelles et les indicateurs de viabilité en fonction de la durée de rétention des ovules in vivo.

\section{RÉSULTATS}

Evolution de la viabilité des ovules après ovulation in vivo et fécondation à différents intervalles de temps

Relations entre les différents indicateurs de viabilité

\section{Juste après ovulation}

Les différents lots d'ovules traités juste après ovulation ont fourni un taux d'éclosion moyen hautement corrélé avec le taux de fécondation, $r^{2}=0,772$. La corrélation entre le taux d'éclosion et le taux de survie embryonnaire est également prononcée $\left(r^{2}=0,601\right)$.

\section{Dans les conditions de stockage in vivo}

Le facteur de corrélation $r^{2}$ est suivi d'un indice indiquant le séjour des ovules dans la cavité abdominale (30,60, 90 et 120 min après ovulation).

Aucune corrélation significative n'a été relevée ni entre les taux de fécondation des ovules fraîchement ovulés et les taux de survie embryonnaires post ovulatoires $\left(r^{2} 30=0,18, r^{2} 60=0,07, r^{2} 90=0,004, r^{2} 120=0,14\right)$, ni entre les taux de fécondation des ovules fraîchement ovulés et les taux d'éclosion post ovulatoires $\left(r^{2} 30=0,09, r^{2} 60=0,03\right.$, $\left.r^{2} 90=0,035, r^{2} 120=0,13\right)$.

Aucune corrélation significative n'a été relevée entre les taux de fécondation post ovulatoires et les taux d'éclosion post ovulatoires correspondants $\left(r^{2} 30=0,3, r^{2} 60=0,08\right.$, $\left.r^{2} 90=0,2, r^{2} 120=0,1\right)$. 
Evolution des taux de viabilité avec la rétention post ovulatoire des ovules et leur relation avec le poids des femelles

Le Tableau I donne les taux moyens de fécondation, de survie embryonnaire et d'éclosion relatifs aux ovules issus des 15 femelles traitées et fécondés à $0,30,60,90$ et 120 min après ovulation.

\section{Tableau I}

Viabilité des œufs (Taux de fécondation, taux de survie embryonnaire et taux d'éclosion) provenant des ovules inséminés après $0,30,60,90$ et $120 \mathrm{~min}$ de rétention post ovulatoire in ovario. Les moyennes (de 15 femelles) suivies par la même lettre ne sont pas significativement différentes.

Table I

Viability of eggs (fertilization rate, embryonic survival rate and hatching rate) from ova fertilized after $0,30,60,90$ and $120 \mathrm{~min}$ of post ovulatory retention in ovario. The means (of 15 females) followed by the same superscript are not significantly different.

\begin{tabular}{|c|c|c|c|c|c|}
\hline Taux de viabilité & $0 \mathrm{~min}$ & $30 \mathrm{~min}$ & $60 \mathrm{~min}$ & $90 \mathrm{~min}$ & $120 \mathrm{~min}$ \\
\hline Taux de fécondation & $70 \pm 13^{a}$ & $78 \pm 12^{a}$ & $70 \pm 12^{a}$ & $67 \pm 14^{a}$ & $63 \pm 13^{a}$ \\
\hline Coefficient de variation & $18,5 \%$ & $15,3 \%$ & $17,1 \%$ & $20,80 \%$ & $20,60 \%$ \\
\hline Taux de survie embryonnaire & $63 \pm 17^{b}$ & $68 \pm 14^{b}$ & $58 \pm 13^{b}$ & $59 \pm 18^{b}$ & $53 \pm 17 b$ \\
\hline Coefficient de variation & $27 \%$ & $20,5 \%$ & $22,4 \%$ & $30,50 \%$ & $32 \%$ \\
\hline Taux d'éclosion & $48 \pm 24^{c}$ & $48 \pm 26^{c}$ & $43 \pm 20^{c}$ & $45 \pm 24^{c}$ & $42 \pm 21^{c}$ \\
\hline Coefficient de variation & $50 \%$ & $54 \%$ & $46 \%$ & $53 \%$ & $50 \%$ \\
\hline
\end{tabular}

Le taux de fécondation a varié entre $70 \% \pm 13$ obtenu juste après ovulation et $63 \% \pm 13$ obtenu 120 minutes après ovulation.

Le taux de survie des embryons issus des ovules fécondés juste après ovulation est de $63 \% \pm 17$. II est de $53 \% \pm 17$ pour les embryons issus des ovules retenus 2 heures après ovulation dans l'abdomen de la femelle.

Les taux d'éclosion moyens des 15 femelles traitées ont varié entre $48 \% \pm 24$ et $42 \% \pm 21$ enregistrés respectivement à 0 et à 120 minutes après ovulation.

Deux heures après ovulation, les taux de fécondation, les taux de survie embryonnaire et les taux d'éclosion différent d'une femelle à l'autre ; les coefficients de variation étant supérieurs à $20 \%(20,6 \%, 32 \%$ et $50 \%$ respectivement) (voir Tableau I).

L'analyse de la variance à un seul facteur (l'Anova I) appliquée pour chaque taux de viabilité des œufs (taux de fécondation, taux de survie embryonnaire et taux d'éclosion) à chaque temps d'extraction des ovules témoigne qu'il n'y a pas eu de différences significatives $(p>0,05)$ entre les taux relatifs aux ovules fécondés juste après ovulation et ceux des ovules fécondés après un séjour prolongé dans la cavité abdominale des femelles (30, 60, 90 et 120 min après ovulation) (voir Tableau I). 
En plus, à partir de 90 minutes de rétention post ovulatoire des ovules, le taux d'éclosion se trouve négativement corrélé au poids des femelles génitrices $\left(r^{2} 90=0,654\right.$; $\left.r^{2} 120=0,568\right)$.

Evolution de la composition des ovules après ovulation in vivo et fécondation à différents intervalles de temps

Les ovules fraîchement ovulés et ceux ayant passé 2 heures dans la cavité abdominale après ovulation ont des poids secs de $32,07 \pm 3,88 \%$ et $32,7 \pm 3,38 \%$ respectivement et des pourcentages de cendres brutes de 4,31 $\pm 0,56 \%$ et 4,38 $\pm 0,36 \%$ (voir Tableau II).

Juste après ovulation, les teneurs des ovules en eau, en fer, en calcium, en protéines totales, en lipides totaux, en phospholipides et en sucres sont de 67,92 $\pm 3,8 \%$, $1,6147 \pm 0,34 \%, 0,189 \pm 0,015 \%, 71,63 \pm 3,4 \%, 10,58 \pm 1,21,6,82 \pm 0,25$ et $3 \pm 0,41$ respectivement. Après un séjour de deux heures après ovulation dans la cavité abdominale, les teneurs sont de 67,19 $\pm 3,36 \%, 1,5923 \pm 0,23 \%, 0,188 \pm 0,017 \%$, $70,45 \pm 3,58 \%, 9,78 \pm 2,31,6,15 \pm 1,15$ et 3,22 $\pm 0,41$ respectivement (voir Tableau II).

L'analyse de la variance à un seul facteur (l'Anova I) appliquée pour chaque composant (eau, poids sec, cendres, fer, calcium, protéines totales, lipides totaux, phospholipides et sucres) à chaque temps d'extraction des ovules témoigne qu'il n'y a pas eu de différences significatives $(p>0,05)$ entre les composants relatifs aux ovules extraits juste après ovulation et ceux relatifs aux ovules ayant passé un séjour prolongé dans la cavité abdominale (30, 60, 90 et 120 min après ovulation) (voir Tableau II).

\section{Tableau II}

Composition des ovules retenus $0,30,60,90$ et 120 min in ovario après ovulation. Les moyennes (de 15 femelles) suivies par la même lettre ne sont pas significativement différentes.

\section{Table II}

The composition of ova retained $0,30,60,90$ and $120 \mathrm{~min}$ in ovario after ovulation. The means (of 15 females) followed by the same superscript are not significantly different.

\begin{tabular}{|l|c|c|c|c|c|}
\hline \multicolumn{1}{|c|}{ Composants } & $\mathbf{0 ~} \mathbf{~ m i n}$ & $\mathbf{3 0} \mathbf{~ m i n}$ & $\mathbf{6 0} \mathbf{~ m i n}$ & $\mathbf{9 0} \mathbf{~ m i n}$ & $120 \mathrm{~min}$ \\
\hline Eau (\% PF) & $67,92 \pm 3,8 \mathrm{a}$ & $67,78 \pm 3,78 \mathrm{a}$ & $68,3 \pm 4,3 \mathrm{a}$ & $67,14 \pm 3,54 \mathrm{a}$ & $67,19 \pm 3,36 \mathrm{a}$ \\
\hline Poids sec (\% PF) & $32,07 \pm 3,88 \mathrm{~b}$ & $32,16 \pm 3,97 \mathrm{~b}$ & $31,62 \pm 4,32 \mathrm{~b}$ & $32,86 \pm 3,68 \mathrm{~b}$ & $32,7 \pm 3,38 \mathrm{~b}$ \\
\hline $\begin{array}{l}\text { Cendres brutes } \\
\text { (\% PS) }\end{array}$ & $4,31 \pm 0,56 \mathrm{c}$ & $4,34 \pm 0,53 \mathrm{c}$ & $4,33 \pm 0,59 \mathrm{c}$ & $4,41 \pm 0,38 \mathrm{c}$ & $4,38 \pm 0,36 \mathrm{c}$ \\
\hline Fer (\% PS x 10-3) & $1,6147 \pm 0,34 \mathrm{~d}$ & $1,60136 \pm 0,25 \mathrm{~d}$ & $1,633 \pm 0,27 \mathrm{~d}$ & $1,60708 \pm 0,25 \mathrm{~d}$ & $1,5923 \pm 0,23 \mathrm{~d}$ \\
\hline Calcium (\% PS) & $0,189 \pm 0,015 \mathrm{e}$ & $0,188 \pm 0,02 \mathrm{e}$ & $0,184 \pm 0,019 \mathrm{e}$ & $0,188 \pm 0,017 \mathrm{e}$ & $0,187 \pm 0,017 \mathrm{e}$ \\
\hline $\begin{array}{l}\text { Protéines totales } \\
\text { (\% PS) }\end{array}$ & $71,63 \pm 3,4 \mathrm{f}$ & $72,26 \pm 3,6 \mathrm{f}$ & $72,23 \pm 3,15 \mathrm{f}$ & $71,3 \pm 3,57 \mathrm{f}$ & $70,45 \pm 3,58 \mathrm{f}$ \\
\hline $\begin{array}{l}\text { Lipides totaux } \\
\text { (\% PS) }\end{array}$ & $10,58 \pm 1,21 \mathrm{~g}$ & $10,17 \pm 1,51 \mathrm{~g}$ & $9,95 \pm 1,81 \mathrm{~g}$ & $9,84 \pm 1,95 \mathrm{~g}$ & $9,78 \pm 2,31 \mathrm{~g}$ \\
\hline $\begin{array}{l}\text { Phospholipides } \\
\text { (\% de lipides totaux) }\end{array}$ & $6,82 \pm 0,25 \mathrm{~h}$ & $6,93 \pm 0,6 \mathrm{~h}$ & $6,64 \pm 0,75 \mathrm{~h}$ & $6,65 \pm 0,87 \mathrm{~h}$ & $6,5 \pm 1,15 \mathrm{~h}$ \\
\hline \begin{tabular}{l} 
Sucres (\% PS) \\
\hline
\end{tabular} & $3 \pm 0,41 \mathrm{i}$ & $3,15 \pm 0,43 \mathrm{i}$ & $3,14 \pm 0,44 \mathrm{i}$ & $3,19 \pm 0,46 \mathrm{i}$ & $3,22 \pm 0,41 \mathrm{i}$ \\
\hline
\end{tabular}


Evolution de la croissance en longueur totale des alevins issus des ovules fécondés à différents intervalles de temps après ovulation

Après avoir passé une semaine dans l'étang, les alevins issus des ovules extraits juste après ovulation atteignent une longueur totale moyenne de 11,34 $\pm 1,02 \mathrm{~mm}$ alors que ceux issus des ovules extraits 120 minutes après ovulation sont longs de $11,9 \pm 0,8 \mathrm{~mm}$ (voir Tableau III).

A 3 semaines après éclosion, les alevins provenant des ovules traités juste après ovulation et ceux issus des ovules traités après 120 minutes de stockage in ovario présentent des longueurs totales de 18,33 $\pm 1,81 \mathrm{~mm}$ et de $20,57 \pm 1,37 \mathrm{~mm}$ (voir Tableau III).

Agés de 4 semaines, les alevins provenant des ovules manipulés juste après ovulation et ceux issus des ovules manipulés 120 minutes après ovulation arrivent à des longueurs totales de 25,72 $\pm 3,1$ et de 25,22 \pm 4,43 mm respectivement (voir Tableau III).

Cinq semaines après éclosion, les ovules fécondés juste après ovulation donnent des alevins longs de $31,09 \pm 2,87 \mathrm{~mm}$. Ceux fécondés après un séjour de 2 heures dans la cavité abdominale procurent des alevins de $30,54 \pm 3 \mathrm{~mm}$ (voir Tableau III).

L'analyse de la variance à un seul facteur (l'Anova I) appliquée pour chaque longueur totale des alevins (L1, L2, L3 et L4) relative à chaque temps d'extraction des ovules montre qu'il n'y a pas eu de différences significatives $(p>0,05)$ entre les longueurs totales des alevins issus des ovules fécondés juste après ovulation et celles des alevins issus des ovules fécondés après un séjour prolongé dans la cavité abdominale $(30,60,90$ et 120 min après ovulation) (voir Tableau III).

\section{Tableau III}

Longueur totale des alevins $(\mathrm{mm})$ issus des ovules fécondés à $0,30,60,90$ et 120 min de rétention post ovulatoire in ovario (L1 = Longueur totale 2 semaines après éclosion, L2 = Longueur totale 3 semaines après éclosion, L3 = Longueur totale 4 semaines après éclosion, L4 longueur totale 5 semaines après éclosion). Les moyennes (de 15 femelles) suivies par la même lettre ne sont pas significativement différentes.

\section{Table III}

The total length of alevins $(\mathrm{mm})$ from ova fertilized after $0,30,60,90$ and $120 \mathrm{~min}$ of post ovulatory retention in ovario ( $L 1=$ total length 2 weeks after hatching, $\mathrm{L} 2=$ total length 3 weeks after hatching, $\mathrm{L} 3=$ total length 4 weeks after hatching, L4 = total length 5 weeks after hatching). The means (of 15 females) followed by the same superscript are not significantly different.

\begin{tabular}{|c|c|c|c|c|c|}
\hline $\begin{array}{l}\text { Longueur totale } \\
(\mathrm{mm})\end{array}$ & $0 \mathrm{~min}$ & $30 \mathrm{~min}$ & $60 \mathrm{~min}$ & $90 \mathrm{~min}$ & $120 \min$ \\
\hline L1 & $11,34 \pm 1,02^{a}$ & $11,74 \pm 1,75^{a}$ & $10,7 \pm 1,17^{a}$ & $11,27 \pm 0,97^{a}$ & $11,9 \pm 0,8^{a}$ \\
\hline L2 & $18,33 \pm 1,81^{b}$ & $18,2 \pm 3,31^{b}$ & $17,88 \pm 2,45^{b}$ & $19,27 \pm 0,66^{b}$ & $20,57 \pm 1,37^{b}$ \\
\hline L3 & $25,72 \pm 3,1^{c}$ & $25,72 \pm 3,1^{c}$ & $27,01 \pm 4,2^{c}$ & $24,18 \pm 3^{c}$ & $25,22 \pm 4,43^{c}$ \\
\hline L4 & $31,09 \pm 2,87^{d}$ & $32,15 \pm 2,84^{d}$ & $33,56 \pm 2,17^{d}$ & $28,38 \pm 1,13^{d}$ & $30,54 \pm 3^{d}$ \\
\hline
\end{tabular}




\section{DISCUSSION}

Les différents lots d'ovules traités juste après ovulation ont fourni un taux d'éclosion hautement corrélé avec le taux de fécondation, $r^{2}=0,772$. Le taux de fécondation s'avère donc lié à la qualité des ovules. Ceci rejoint les résultats de plusieurs auteurs qui avancent que le taux de fécondation est un critère de qualité des œufs si il est étroitement corrélé au taux d'éclosion. Un taux d'éclosion satisfaisant témoigne d'une bonne qualité d'œufs (SPRINGATE et al., 1985 ; SRIVASTAVA et BROWN, 1991 ; BRUCE et al., 1993 ; FERNANDEZ-PALACIOS et al., 1995, 1997 ; LARSON et al., 1997).

Cette dernière serait attribuée à la richesse éventuelle des ovules en acides gras saturés et insaturés. Chez bon nombre de poissons, ces composés semblent être essentiels pour l'accomplissement de la fécondation et l'initiation de l'embryogenèse (SHIMMA et al., 1978 ; PARRISH et al., 1994 ; WIEGAND, 1996 ; TOCHER et al., 1996 ; NAVAS et al., 1997).

Cependant, chez Cyprinus carpio, Rutilis rutilis et 0 . mykiss, des résultats antérieurs contradictoires aux nôtres ont été rapportés ; le taux de fécondation n'étant pas relié à la qualité des ovules en terme de taux d'éclosion (ZONOVA, 1973 ; SAVOSTYANOVA et NIAKANDROV, 1976 ; KOKUREWICAZ, 1981).

La corrélation entre le taux d'éclosion et le taux de survie embryonnaire est également prononcée $\left(r^{2}=0,601\right)$. En effet, une fois que l'embryon a dépassé le stade morula, le risque d'avortement diminue. La mortalité massive si elle a lieu, prend place aux premiers stades du développement embryonnaire. Ce résultat semble être en concordance avec les travaux de CRAIK et HARVEY (1984) et SPRINGATE et al., (1985) qui ont approché cet aspect chez la truite arc en ciel. Cependant, nos résultats ne sont pas analogues à ceux de KATO et KAMLER (1983) qui n'ont relevé aucune corrélation entre les capacités de l'embryon à survivre pendant les premières et les dernières phases du développement embryonnaire chez Salmo gairdneri.

Quand il s'agit de vieillissement ovocytaire, un bon taux de fécondation des ovules traités juste après ovulation n'implique pas des taux de survie embryonnaire et des taux d'éclosion satisfaisants quand ces ovules séjournent dans l'abdomen du poisson pendant différentes périodes après ovulation (30,60, 90 et $120 \mathrm{~min}$ ). En effet, aucune corrélation significative n'a été relevée entre les taux de fécondation des ovules fraîchement ovulés d'une part et les taux de survie embryonnaire et les taux d'éclosion post ovulatoires d'autre part. II ressort de ce qui précède que quoique le taux de fécondation soit un paramètre prédictif de la qualité des ovules, il n'est pas un indicateur de leur tolérance au vieillissement in vivo.

Dans le même contexte, même si les ovules fécondés 30,60 , 90 et 120 min après ovulation fournissent d'excellents taux de fécondation, cela n'implique pas forcément des taux de survie embryonnaire et des taux d'éclosion correspondants satisfaisants. La qualité des ovules serait fortement impliquée. En effet, aucune corrélation significative n'a été enregistrée entre les taux de fécondation post ovulatoires d'une part et les taux de survie embryonnaire et les taux d'éclosion correspondants d'autre part.

II s'avère donc que quoique les paramètres vitaux utilisés lors de notre étude donnent une idée sur la qualité des ovules, ils ne sont pas très suffisants. C'est pourquoi on a eu recours à d'autres paramètres plus pertinents (quelques aspects sélectifs de la composition des ovules).

Deux heures après ovulation, les taux de viabilité ont montré une grande variabilité entre les différentes femelles reproduites au cours de la même saison ou au cours des différentes saisons ; les coefficients de variation calculés pour les taux de viabilité relatifs 
à deux heures de rétention ont dépassé $20 \%$. Ces résultats indiquent que les ovules de la carpe herbivore ont une tolérance très variable aux conditions de stockage in vivo. Une telle tolérance serait en rapport avec leur qualité et leur composition biochimique. La composition biochimique des ovules des poissons étant tributaire des statuts nutritionnel et héréditaire (LAVENS et SORGELOOS, 1991 ; CERDA et al., 1995 ; HARREL et WOODS, 1995 ; FERNANDEZ-PALACIOS et al., 1995 ; VERAKUNPIRIYA et al., 1996 ; BELL et al., 1997) et affectant considérablement la viabilité des œufs et la survie de la progéniture (BLOM et DABROWSKI, 1995, 1996 ; CERDA et al., 1994, 1995 ; FERNADEZPALACIOS et al., 1995, 1997 ; RAINUZZO et al., 1997).

Outre cela, il s'est avéré lors de cette étude que la viabilité post ovulatoire des ovules retenus in vivo semble être conditionnée par la qualité des ovules fournis. Les résultats ont montré que des ovules de bonne qualité fournissent des taux satisfaisants de fécondation, de survie embryonnaire et d'éclosion même s'ils sont fécondés 120 min après ovulation alors que des ovules de mauvaise qualité donnent des taux médiocres même s'ils sont inséminés juste après ovulation. Ces résultats sont similaires à ceux de BILLARD et al. (1986), à ceux de DROUSSI $(1989,1998)$ et à ceux de LINHART et BILLARD (1995) qui suggèrent que la survie post ovulatoire des ovules retenus in vitro ou in vivo est tributaire de la qualité des ovules chez la carpe commune, chez la carpe argentée et chez le silure glane.

Les femelles manipulées pendant les trois compagnes de reproduction (1996, 1997 et 1998) n'ont ni le même âge ni le même poids (3 à 5,6 kg). On n'a pas relevé l'âge exact des femelles mais il s'est avéré lors de cette étude que le poids des femelles conditionne la viabilité post ovulatoire des ovules à partir de 90 minutes de rétention in ovario. En effet, la qualité des œufs des poissons est considérablement affectée par l'âge et le poids des géniteurs (BAGENAL, 1971 ; KAMLER, 1976 ; KUZNETSOV et KHALITOV, 1979 ; PARRISH et al., 1995, 1996 ; EVANS et al., 1996). Donc, ces facteurs maternels pourraient être mis en cause dans la disparité de la qualité et de la viabilité post ovulatoire des ovules observées au cours de nos expériences; les femelles manipulées produiraient alors des ovules qui seraient biochimiquement différents. L'hypothèse formulée est d'ailleurs confirmée par les résultats d'analyses. On a noté une grande variabilité entre les femelles pour tous les composés examinés (eau, poids sec, cendres brutes, fer, calcium, sucres, protéines totales, lipides totaux et phospholipides) $(p<0,05)$, les femelles fournissent effectivement des ovules biochimiquement différents.

En conclusion, on note une large variation dans la viabilité post ovulatoire (TF, TSE, TE) des différents lots d'ovules issus des différentes femelles malgré qu'ils aient été extraits, fécondés et incubés sous des conditions identiques et malgré que ces lots soient obtenus à partir des géniteurs traités uniformément, particulièrement à l'égard de la nourriture.

Les résultats ont montré que la rétention des ovules de la carpe herbivore in vivo reste sans effet sur le taux de fécondation, le taux de survie embryonnaire et le taux d'éclosion, et ce pour un intervalle de temps allant jusqu'à 2 heures après ovulation lorsque les ovules sont de bonne qualité. Similairement à nos résultats, chez Limanda yokohama, chez Plecoglossus altivelis, chez Clarias macrocephalus, chez Silurus glanis, et chez Heterobranchus longifilis, le taux d'éclosion des ovocytes ovulés retenus dans l'ovaire demeure constant pendant les deux heures suivant l'ovulation (HIROSE et al., 1977, 1979 ; MOLLAH et TAN, 1983 ; LINHART et BILLARD, 1995 ; LEGENDRE et OTÉMÉ, 1995). II n'en est pas de même pour les œufs de la perche rayée Roccus saxatilis qui gardent leur viabilité au maximum pour 1 heure (STEVENS, 1966). Au cours de nos expériences, on a évalué la tolérance au vieillissement in vivo en minutes. En effet, chez les cyprinidés, le vieillissement in vivo s'avère plus rapide que celui des salmonidés (HORVATH, 1978a ; SUZUKI, 1980 ; CRAIK et HARVEY, 1984). 
Nos résultats présentent une similitude avec ceux de SUZUKI (1980), JÄHNICHEN (1981), MARCEL (1981) et DROUSSI (1989). Ces auteurs avancent que le taux de fécondation des ovules de la carpe retenus dans la cavité abdominale reste constant pour des intervalles de temps allant de 2 à 6 heures de rétention post ovulatoire alors que HORVATH (1978b) range la survie post ovulatoire (in vivo) de ces ovules entre 50 et 80 minutes seulement.

Les analyses des aspects sélectifs de la composition des ovules (eau, poids sec, cendres, calcium, fer, sucres, protéines totales, lipides totaux et phospholipides) ont indiqué qu'il n'y a pas eu de changements majeurs associés au phénomène de vieillissement in vivo. Ainsi, il n'y a pas eu de perte de matière sèche, ni augmentation de l'eau. Ceci soulignerait l'étendue du rôle de la membrane plasmique dans la limitation des flux transmembranaires de l'eau (ALDERDICE, 1988). Le fait que 2 heures après ovulation, la teneur des ovules en protéines totale reste constante pourrait être interprété par l'absence de rupture protéolytique des protéines du vitellus et l'absence de perte à travers les membranes des ovules de petites molécules organiques tels que les acides aminés et les peptides. Le fait qu'il n'y aurait pas eu de rupture protéolytique des macromolécules organiques implique l'absence d'augmentation du contenu en osmolytes. II s'ensuivrait alors une absence de gonflement osmotique et d'augmentation de la teneur des ovules en eau. La constance des teneurs des ovules en fer et en calcium après ovulation enregistrée lors de cette étude dénoterait l'absence des changements dégénératifs du vitellus. Ces résultats sont comparables à ceux de CRAICK et HARVEY (1984) qui ont travaillé sur les mêmes constituants (sauf phospholipides et sucres) chez la truite arc en ciel pour une période de 18 jours après ovulation.

Durant le séjour des ovules dans la cavité ovarienne, les ovules de la carpe herbivore ont donc gardé leur composition constante. Ils se seraient bien conservés. Or, la composition biochimique reflète les demandes embryonnaires et larvaires du point de vue nutrition et croissance (KJORSVICK et al., 1990). Ceci expliquerait que la rétention des ovules au sein de l'abdomen reste sans effet sur leur viabilité subséquente et sur le développement des alevins en terme de croissance en longueur totale du moins jusqu'à 5 semaines après éclosion.

Néanmoins, la constance de la composition des ovules et la maintenance de la capacité de développement des œufs et des alevins deux heures après ovulation, notée chez la carpe herbivore ne rend pas compte de leur réaction vis à vis d'un stockage in vivo plus prolongé. En effet, les résultats de SAKAl et al., (1975) ; SUZUKI (1975) ; HIROSE et al., (1979) ; STATOVA et al., (1982) ; CRAIK et HARVEY (1984) ; BILLARD et al., (1986); LINHART et BILLARD (1995) ont montré chez d'autres poissons que le vieillissement ovocytaire poussé se traduit aussi bien par une baisse du taux de fécondation que par des mortalités embryonnaires plus ou moins tardives. La fragilisation des progénitures issues des pontes surmatures n'est, dès lors, pas à exclure.

La prolongation de la viabilité des ovules enregistrée lors de cette étude pourrait être régulée par un contrôle hormonal. En analogie avec les autres vertébrés, CRAIK et HARVEY (1984) suggèrent que les follicules post ovulatoires peuvent agir comme organes endocriniens produisant des hormones qui maintiennent la viabilité des œufs dans leur voisinage, en l'occurrence, la cavité abdominale. En effet, BILLARD et ses co-auteurs (1986) ont affirmé que les ovules dégénèrent plus rapidement et ont un taux de fécondation moindre quand ils sont maintenus in vitro qu'in vivo.

En outre, l'activité respiratoire pourrait être mise en cause pour expliquer le phénomène de la prolongation de la viabilité des œufs et l'absence d'effet de rétention sur la composition de l'ovule et sur la croissance des alevins en longueur totale observée lors de nos expériences. En effet, concernant la carpe herbivore, GOSH (1985) rapporte que les taux respiratoires les plus élevés des ovules sont observés après stripping et ne 
diminuent de $50 \%$ qu'après 5 à 6 heures de rétention dans la cavité ovarienne à la même température. En plus, l'augmentation de la disponibilité en oxygène prolonge la viabilité des ovules stockés in vivo (JENSEN et ALDERDICE, 1984).

Par ailleurs, la variabilité de la tolérance au vieillissement enregistrée entre les femelles au cours de nos expériences, pourrait incomber au fait que les ovocytes ovulés n'auraient pas des taux respiratoires similaires puisqu'elles proviennent des femelles n'ayant pas le même âge. Effectivement, ZHUKINSKIY et GOSH $(1970,1974)$ et GOSH (1985) ont relié la croissance maternelle au niveau d'activité respiratoire des ovules.

Dans ce contexte, il est intéressant de souligner le rôle éventuel les caroténoïdes dans la respiration des ovules de la carpe herbivore pendant leurs stockage in vivo. En effet, au cours de nos expériences, les ovules sont maintenus dans la cavité ovarienne sans approvisionnement direct en sang et probablement avec une disponibilité moindre en oxygène. En effet, GOSH (1981) a montré que le taux respiratoire est d'un niveau plus bas dans les ovocytes ovulés que dans les ovocytes intrafolliculaires. La possibilité que les caroténoïdes agissent comme réserve intracellulaire d'oxygène et assistent la respiration sous des conditions où l'oxygène serait déficient, pourrait alors être évoqué utilement. Les ovules de carpe herbivore à teneur substantielle de caroténoïdes seraient susceptibles de survivre plus longtemps avant d'être surmatures et non viables.

Cette hypothèse est compatible avec les résultats de DROUSSI (1998) qui a montré que les ovules de la carpe herbivore les plus pigmentés fournissent des taux satisfaisants de fécondation et de survie embryonnaire comparés aux ovules moins pigmentés.

En plus, la tolérance des ovules de la carpe herbivore au stockage in vivo au bout de deux heures pourrait être dû au fait que ces cellules accumulées dans la cavité ovarienne en contact avec le fluide ovarien seraient sujettes à une régulation osmotique et ionique. Par le biais de ce contrôle, les ovocytes ovulés de la carpe herbivore conserveraient leur propre intégrité et par la suite mèneraient à bien le développement embryonnaire et larvaire subséquent. En effet, chez les poissons Téléostéens, cette activité régulatrice est assurée par la membrane plasmique de l'ovule et par la limitation des flux transmembranaires de l'eau et des ions (ALDERDICE, 1988).

\section{CONCLUSIONS ET PERSPECTIVES}

Le présent travail nous a permis de formuler les conclusions suivantes :

- Les ovules de la carpe herbivore peuvent être maintenus dans la cavité ovarienne pendant 2 heures, tout en procurant des taux satisfaisants de fécondation, de survie embryonnaire et d'éclosion s'ils sont de bonne qualité. Ceci représente un avantage pratique considérable pour l'opérateur en écloserie. La contrainte du temps n'est pas assez sévère entre le moment de l'ovulation et celui de la récolte des ovules en vue de la reproduction artificielle.

- Au regard de ces résultats il s'avère que chez la carpe herbivore, cette maintenance de viabilité des œufs est femelle-dépendante. En effet, à partir de 90 minutes de rétention post ovulatoire des ovules in ovario, le poids des femelles s'avère négativement corrélé au taux d'éclosion.

- Les ovules n'ont pas été sujets à des changements majeurs en ce qui concerne les constituants suivants : poids sec, teneur en eau, cendres, calcium, fer, protéines totales, lipides totaux, phospholipides et sucres. Ces résultats d'analyses de laboratoire sont très compatibles avec les données des paramètres biologiques déterminés en 
écloserie puisque, après un séjour de 2 heures dans la cavité ovarienne, les ovules de bonne qualité ne subissent pas des changements affectant leur viabilité.

- Le développement des alevins en longueur totale jusqu'à 5 semaines après éclosion ne semble pas être altéré par la rétention des ovules dans la cavité abdominale deux heures après ovulation. Cette conclusion confirmerait les résultats des aspects sélectifs de la composition étudiée.

Dans ce contexte, nous prévoyons d'examiner les aspects étudiés de la composition de l'ovule et d'autres (acides gras, caroténoïdes, sodium, potassium, phosphore) en prolongeant la durée du séjour des ovules dans la cavité abdominale. Ceci nous permettrait l'établissement éventuel d'un ou des marqueurs biochimiques du vieillissement in vivo des ovules.

\section{BIBLIOGRAPHIE}

ALDERDICE D.F., 1988. Osmotic and ionic regulation in Teleost eggs and larvae. In : Fish Physiology, Part A: Eggs and Larvae, Vol XI, Acad. Press, HOAR W.S. and RANDALLS D.J. Eds., 545 p.

A.O.A.C. 1970. Official method of analysis. Association of Official Analytical Chemist, Washington, DC, $11^{\text {th }}$ ed.

BAGENAL T.B., 1971. The interrelation of the size of fish eggs, the date of spawning and the productive cycle. J. Fish Biol., 3, 207-219.

BARTLETT G.R., 1959. Posphorus assays in column chromatography. J. Biol. Chem., 234, 464-466.

BELL J.G., FARANDALE B.M., BRUCE M.P., NAVAS J.M., CARILLO M., 1997. Effect of broodstock dietary lipid on fatty acid composition of eggs from sea bass (Dicentrarchus labrax). Aquaculture, 149, 107-119.

BILLARD R., GATTY J.L., HOLLEBECQ M.G., MARCEL J., SAAD A., 1986. Biology of gametes, eggs and embryos. In : Aquaculture of Cyprinids, INRA, Paris, 1986, BILLARD R. et MARCEL J. Eds., 502 p.

BLOM J.H., DABROWSKI K., 1995. Reproductive success of female rainbow trout, Oncorhynchus mykiss in response to graded dietary ascorbyl monophosphate levels. Biol. Reproduct., 52, 1073-1080.

BLOM J.H., DABROWSKI K., 1996. Ascorbic acid metabolism in fish: is there a maternal effect on the progeny? Aquaculture, 147, 215-224.

BRUCE M.P., SHIELDS R.J., BELL M.V., BROMAGE N.R., 1993. Lipid class and fatty acid composition of eggs of Atlantic Halibut (Hippoglossus hippoglossus) in relation to egg quality in captive broodsock. Aquaculture and Fisheries management, 24 (3), 417-422.

CERDA J., CARRILLO M., ZANY S., RAMOS J., 1994. Effect of food ration on estrogen and vitellogenine plasma levels, fecundity and larval survival in captive sea bass (Dicentrarchus labrax). Preliminary observations. Aquat. living Resour., 7 (4), 225-266.

CERDA J., CARRILLO M., ZANY S., RAMOS J., SERRANO R., 1995. Short and long term dietary effect on female sea bass (Dicentrarchus labrax): Seasonal changes in plasma profiles of lipids and sex steroides in relation to reproduction. Comp. Biochem. Physiol. (C) ), 111, 83-91.

CRAIK J.C.A., HARVEY S.M., 1984. Egg quality in rainbow trout: the relation between egg viability, selected aspects of egg composition, and time of stripping. Aquaculture, 40, 115-134.

DROUSSI M., 1989. Effect of temperature and pure oxygen on the storage and viability of infertilized common carp eggs. Auburn University of Alabama, Master of Sciences, $78 \mathrm{p}$. 
DROUSSI M., 1998. Qualité des ovules des carpes chinoises. In : Les Actes du $3^{\text {ème }}$ Congrès National de l'Association Marocaine de Limnologie (A.M.L.) sous le thème: Les eaux continentales et le développement durable. Oujda, les 23-24-25 avril 1998, $200 \mathrm{p}$.

DUBOIS M., GILLES G.A., HAMILTON J.K., REBELS P.A., SMITH F., 1956. Colorimetric methods for determination of sugars and related substances. Anal. Chem., 226, 497-509.

EVANS R.P., PARRISH C.C., BROWN J.A., DAVIS P.J., 1996. Biochemical composition of eggs from repeat and first-time spawning captive Atlantic Halibut, (Hippoglossus hippoglossus). Aquaculture, 139, 139-149.

FERNANDEZ-PALACIOS H., IZQUIERDO M.S., ROBAINA L., VALENCIA A., SALHI M., VERGARA J.M., 1995. The effect of n-3 HUFA in broodstock diets on egg quality of gilthead seabream (Sparus aurata). Aquaculture, 132, 325-337.

FERNANDEZ-PALACIOS H., IZQUIERDO M.S., ROBAINA L., VALENCIA A., SALHI M., MONTERO D., 1997. The effect of dietary protein and lipid from squid and fish meals on egg quality of broodstock for gilthead seabream (Sparus aurata). Aquaculture, 148, 233-246.

FOLCH J., LEES M., SLOANE STANLEY G.H.S., 1957. A simple method for the isolation and purification of total lipids from animal tissues. J. Biol. Chem., 226, 497-509.

GOSH R.I., 1981. Intensity of energetic metabolism in oocytes and maturate eggs of roach and bream. In : Ontogenetic Diversity in Fish. Naukova Dumka. Kiev, 147 p.

GOSH R.I., 1985. Energy metabolism of fish reproductive cells and embryos. In : Ontogenetic Diversity in Fish. Naukova Dumka. Kiev, 147 p.

HARREL R.M., WOODS L.C., 1995. Comparative fatty acid composition of eggs from domesticated and wild striped bass (Morone saxatilis). Aquaculture, 133, 225-233.

HIROSE K., ISHIDA R., SAKAI K., 1977. Induced ovulation of ayu using Human Chorionic Gonadotropin (HCG), with special reference to changes in several characteristics of eggs retained in the body cavity after ovulation. Bull. Jpn. Soc. Sci. Fish., 43, 409-416.

HIROSE K., MACHIDA Y., DONALDSON E.M., 1979. Induced ovulation of Japanese flounder (Limanda Yokohamae) with human chorionic gonadotropin and salmon gonadotropin, with special reference to changes in quality of eggs retained in the body cavity after ovulation. Bull. Jpn. Soc. Sci. Fish., 45, 31-36.

HORVATH L., 1978a. Relation between ovulation and water temperature by farmed Cyprinids. Aquacult. Hung. (Svarvas), 1, 58-65.

HORVATH L., 1978b. Experience in propagation of the common carp (Cyprinus carpio), out of the spawning season. Aquacult. Hung. (Szarvas), 1, 66-72.

JAHNICHEN H., 1981. Kurzzeitkonservierung von Karpfensperma als weitere Möglichkeit der Rationalisierung der kunstlichen Vermehrung von Karpfen (Cyprinus carpio L.). Z. Binenfischerei DDR, 28, 244-245.

JENSEN J.O.T., ALDERDICE D.F., 1984. Effect of temperature on short-term storage of eggs and sperm of Chum Salmon (Oncorhynchus keta). Aquaculture, 37, 251-265.

KAMLER E., 1976. Variability of respiration and body composition during early developmental stages of carp. Pol. Arch. Hydrobiol., 30, 431-485.

KATO T., KAMLER E., 1983. Criteria for evaluation of fish egg quality as exemplified for Salmo gairdneri. Bull. Nat. Res. Inst. Aquaculture, 4, 61-78.

KJORSVIK E., MANGOR-JESEN A., HOLMFJORD I., 1990. Egg quality in fishes. Adv. Mar. Biol., 26, 71-113.

KOKUREWICAZ B., 1981. Effect of different thermal regimes on reproductive cycle of tench (Tinca tinca L.). Part VII. Embryonal development of progeny. Pol. Arch. Hydrobiol., 28, 234-256.

KUZNETSOV V.A., KHALITOV N.K., 1979. Alteration in fecundity and egg quality of Rutilis rutilis, in connection with different feeding conditions. J. Ichtyol., 18, 63-70.

LARSON D.G.J., MYLONAS C.C., ZOHAR Y., CRIM L.W., 1997. Gonadotropin-releasing hormone analogue ( $\mathrm{Gn} \mathrm{RH}-\mathrm{A})$ induces multiples ovulation of high quality 
eggs in a cold water, batch spawning teleost, the yellowtail flounder, (Pleuronectes ferrugineus). Can. J. Fish. Aquat. Sci., 54, 957-1964.

LAVENS P., SORGELOOS L.P., 1991. Variation in egg and larval quality in various fish and crustacean species. Larvi'91., Fish and Crustacean Larviculture. Symposium LAVENS P., SORGELOOS L.P., OLLEVIER E.F. (Eds.), E.A.S. Spec. publ., 15, 221-222.

LEGENDRE M., OTÉMÉ Z.J., 1995. Effect of varying latency period on the quantity and the quality of ova after HCG induced ovulation in the African catfish, Heterobranchus longifilis (Teleostei, Clariidae). Aquat. Living Resour., 8, 309-316.

LINHART O., BILLARD R., 1995. Survival of ovulated oocytes and ova in the european catfish, Silurus glanis after in vivo and in vitro storage or exposure to various solutions. Aquat. Living Resour., 8, 317-322.

MARCEL J., 1981. Contrôle de la reproduction et gestion des gamètes de quelques espèces de poissons téléostéens. Ecole Pratique des Hautes Etudes. $8^{\text {ème }}$ Section. Diplôme, 133 p.

MOLLAH M.F.A., TAN E.S.P., 1983. Viability of catfish, (Clarias macrocephalus, Gunther) eggs fertilized at varying post ovulation times. J. Fish Biol., 22, 563-566.

NAVAS J.M., BRUCE M., TRRUSH M., FARNDALE B.M., BROMAGE N., ZANUY S., CARRILO M., BELL J.G., RAMOS J., 1997. The impact of seasonal alteration in the lipid composition of broodstock diet on egg quality in the european sea bass. J. Fish Biol., 51, 760-773.

PARRISH C.C., CASTELL J.D., BROWN J.A., BOSTON L., STRICKLAND J.S., SOMERTON D.C., 1994. Fatty acid composition of Atlantic halibut eggs in relation to fertilization. Bull. Aquacult. Assoc., 94 (2), 36-38.

PARRISH C.C., YOUNG Z., WELL J.S., CASTELLE J.D., BROWN J.A., 1995. Fatty acid composition of eggs of captive Atlantic halibut, (Hippoglossus hippoglossus) in relation to larval survival. E.A.S. Spec. Publ., 24, 30-33.

PARRISH C.C., YOUNG Z., BROWN J.A., 1996. Triacylglycerol composition of eggs from captive Atlantic halibut over the spawning saison. Bull. Aquacult. Asso. Canada, 1, 41-43.

RAINUZZO J.R., REITAN K.I., OLSEN Y., 1997. The significance of lipids at early stages of marine fish: a review. Aquaculture, 155, 103-115.

SAKAI K., NOMURA M., TAKASHIMA F., OTISHI H., 1975. The over-ripening phenomenon of rainbow trout. II. Changes in the percentage of eyed eggs, hatching rate and incidence of abnormal alevins during the process of over-ripening. Bull. Jpn. Soc. Sci. Fish., 41, 855-860.

SAVOSTYANOVA G.C., NIAKANDROV V.Ya., 1976. Dependance of some biometrical indices of eggs on age of the rainbow trout females. Izv. gos. Nauchno-issled. Inst. Ozernogo i rechnogo Rybnogo Khoz., 133, 3-7.

SHIMMA Y.T., KATO T., FUKUDA Y., 1978. Effect of feeding methanol grown yeast on growth and maturity of yearling rainbow trout and egg hatchability. Bull. Freshwat. Fish. Res. Lab., 28, 21-28.

SPRINGATE J.R.C., BROMAGE N.R., CUMARARANATUNGA P.R.T., 1985. The effect of different rations on fecundity and egg quality in the rainbow trout (Salmo gairdneri). In: Nutrition and Feeding in Fish. Acad. Press, London. COWEY C.W., MACKIE A.M. and BELL J.G. (Eds.), 371-393.

SRIVASTAVA R.K., BROWN J.A., 1991. The biochemical characteristics and hatching performance of cultured and wild Atlantic salmon (Salmo salar) eggs. Can. J. Zool., 69, 2436-2441.

STATOVA M.P., TALIKNA M.G., KALINICH R.A., 1982. Physiological-chemical characteristics of the eggs of common carp (Cyprinus carpio) (Cyprinidae) under conditions of fish farming. J. Ichtyol., 22, 117-128.

STEVENS R.E., 1966. Hormone-induced spawning of striped bass for reservoir stocking. Prog. Fish. Cult., 28, 19-28.

SUZUKI R., 1975. Duration of development capacity of eggs after ovulation in the loach, Cyprinid fish. Aquaculture, 23, 92-99. 
SUZUKI R., 1980. Duration of developmental capability of carp eggs after ovulation in the ovarian cavity. Bull. Nat. Res. Inst. Aquacult., 1, 1-6.

TOCHER D.R., DICK J.R., SARGENT J.R., 1996. Stimulation of proliferation of an essential fatty acid-deficient fish cell line by $\mathrm{C}-20$ and C-22 polyunsatured fatty acid and effects on fatty acid composition. Prostaglandins Leukotrienes and Essential Fatty Acids, 55 (5), 345-356.

VERAKUNPIRIYA V., WATANABE T., MUSHIAKE K., KIRON V., SATOH S., TAKEUCHI T., 1996. Effect of broodstock diet on the chemical components of milt and eggs produced by yellowtail. Fisheries Sciences, 186, 110-69.

WIEGAND M.D., 1996. Utilization of yolk fatty acids by goldfish embryos and larvae. Fish. Biol. Biochem., 15, 21-27.

ZHUKINSKIY V.N., GOSH R.I., 1970. Viability of embryos in dependence of respiratory rate ofovulated egg in roach and bream of different ages. Gidrobiol. Zh., 6 (4), 60-70.

ZHUKINSKIY V.N., GOSH R.I., 1974. Viability of embryos in dependence of rate of energetic metabolism in ovulated egg and sperm of roach and bream of early age. In : Ontogenetic Diversity in Fish. Naukova Dumka, Kiev, $147 \mathrm{p}$.

ZHUKINSKIY V.N., KIM Y.D., 1980. Characteristics of age related - variability in the composition of amino acids and lipids in mature and overripe eggs of the Azov roach (Rutilis rutilis heckeli), and the bream (Abramis brama). J. Ichtyol., 20, 121-132.

ZONOVA A.S., 1973. Relation between the egg size and some properties of female carps (Cyprinus carpio L). Vopr. Ikhtiol., 13, 816-825. 
